\title{
Research on the Mode of Efficaciously Solving "Agricultural Super-Docking" Development Difficulties in China
}

\author{
Guangshu Xu, Haifang Zhang, Xuewen Zhang \\ Graduate Faculty, Beijing Wuzi University, Beijing, China \\ Email: 13716996816@163.com
}

Received 4 August 2016; accepted 20 August 2016; published 23 August 2016

Copyright (C) 2016 by authors and Scientific Research Publishing Inc.

This work is licensed under the Creative Commons Attribution International License (CC BY). http://creativecommons.org/licenses/by/4.0/

(c) (i) Open Access

\section{Abstract}

Constructing circulation mode of high feasibility and good implementation effect is the key method to solve difficulties in the development of "Agricultural Super-Docking" at present in China. There are many problems that exist in the process of the development of "Agricultural SuperDocking" by our analyzing from both the benefit of subjects and the whole supply chain perspectives. A large amount of relevant literature research shows that adopting the "cooperation between the government and the third party logistics enterprises to build distribution center" innovation model can solve these problems. This model can effectively remove obstacles between farmers and supermarkets. It is conducive to the development of small and medium-scale the farmer cooperatives, supermarkets and the third party logistics enterprises that don't have a strong financial strength and a high degree of brand influence. In addition, it can also produce considerable social benefits. In order to ensure the implementation efficiency of the model to maximize, some management issues in the implementation process about the determination of core subject in supply chain, the method of evaluating the third party logistics enterprises grade, the planning of the number of customers for the distribution center, the mechanism of income distribution among multiple subjects and the implementation of relevant policies after the model into practice should be taken into account.

\section{Keywords}

Agricultural Super-Docking, Distribution Center, The Government and the Third Party Logistics Enterprises, Quality and Safety

\section{Introduction}

People's demand for agricultural products is rigid and space-time unbiased characteristics. Therefore, agricul-

How to cite this paper: Xu, G.S., Zhang, H.F. and Zhang, X.W. (2016) Research on the Mode of Efficaciously Solving "Agricultural Super-Docking" Development Difficulties in China. World Journal of Engineering and Technology, 4, 460-468. 
ture is the source of food and clothing and the foundation of life for a region and even a country, which is also significant for the development of the whole national economy. In order to solve the problem of circulation of agricultural products, China's State Council and the Ministry of Agriculture jointly issued the "Circular on the pilot work of agricultural super-docking" in 2008, and began the deployment of the pilot work.

But the outcome of "agriculture super-docking" practice of many years is not satisfactory in China due to the implementation of many of the problems in the process, and the recent fresh business as an emerging model of agricultural products circulation was widespread concern in the community. Some scholars already have put forward that electronic commerce has the inherent mechanism to break deadlock of agricultural products circulation. But fresh electricity supplier is still in its infancy from the overall development of the China. With the exception, a few first-tier cities can do a wide range of coverage; the other consumers of two- or three-tier cities still need to buy fresh agricultural products from the supermarket and some retail locations of these entities. And it is very difficult for consumers to change the traditional consumption habits of instant consumption and picking purchase for fresh products as well. And the most important is that the e-commerce operation of fresh agricultural products has not yet established the perfect quality traceability system in China. If there are quality and safety problems, virtual character of e-commerce will make the process of consumer rights protection become very difficult. It also determines that there will be a considerable part of the consumers who will continue to choose this supermarket channels which have responsible party of some entity to purchase fresh agricultural products in a long time.

So it is very important to construct a practical mode of circulation to break the current "agricultural superdocking" development dilemma in China. This paper combines the problems of "agriculture super-docking" and a lot of theoretical research to put forward and build an innovative docking mode as well as discusses detailedly the management countermeasures and suggestions from various aspects in the implementation of the model. It not only provides a theoretical reference for many economic subjects in the agricultural products circulation, but also has a great significance to improve social benefits, such as stabilizing the price of agricultural products, balancing the supply and demand of the agricultural market and ensuring the most basic survival and safety of all the people.

\section{Development Dilemma and Related Research}

From the perspective of the main benefits of "agriculture super docking", due to the lack of perfect benefit docking mechanism with real contract in China, farmers has been at a weak position in the docking system and income is not improved as well, so their enthusiasm to participate in the docking is not high [1]. The purpose that many supermarkets operate fresh produce is to attract more consumers to come to shopping, if the supermarket chain size does not reach a certain degree, the cost of the establishment of fresh logistics distribution center to achieve a better docking will affect the efficiency of the supermarket [2]. Therefore, most of the small and medium-sized supermarkets and even large supermarket chains have chosen large wholesale market which is a traditional mode of circulation as the main source of fresh agricultural products. Although the quantity of many of the third party logistics companies that responsible for the cold chain transportation in the docking process is very large in Chain, their scale is small. Their financial strength is limited, and the lack of effective cooperation between regions makes cold chain infrastructure have yet to be integrated. So they aren't able to provide a comprehensive integrated the whole process cold chain service for fresh agricultural products [3]. Eventually, it led to the rate of fresh product damage is high and the decline in customer satisfaction, but also made the third party logistics enterprise's reputation and effectiveness was influenced. And the government's support policy for cold chain logistics enterprises fell into the "rolling snowball effect", which is the first stage of subsidies make impairment products target market to increase the supply, and lead to second stage impairment product prices fell, finally the value of the subsidy has a gradual increase. With the diminishing marginal effect, the policy effect for promoting the construction of cold chain logistics system became weaker, so government spending has not obtained the ideal social benefits [4].

From the perspective of the whole "agriculture super docking" supply chain, the main problem is the quality and safety of agricultural products, although "agriculture super-docking” can reduce the risk of food safety problems in a certain extent because of reduction of circulation link compared to traditional circulation pattern of agricultural products. In fact, many supermarkets have no more willingness to focus on controlling incoming quality work strictly, and because some individual food quality and safety inspection agencies management is 
not good, resulting in false certification made docking products still exist great security hidden danger [5]. Consumers began to doubt the quality of docking products, which greatly affected the further promotion of agricultural super-docking; Followed by the standard of agricultural products, on the one hand, farmers lack guidance about the unified professional cultivation technology resulting in uneven product quality. In terms of size, weight, appearance, taste and so on, products lack a high grade division standard recognized by the market highly, so it's difficult to achieve "good products correspond to high prices", thus farmers cannot get positive incentives. On the other hand, most of the products offered by the farmers are primary agricultural products which have low added value, the products sold to consumers directly by supermarkets have a high added value, most of which are processed by standard, so the two sides cannot achieve good docking in circulation and processing standard. Finally is the policy environment problem, at present, many relevant policies the government promulgated aren't mandatory for “agriculture super-docking” and their implement effect is poor. For example Xinghuo livestock cooperatives established in 2007, it supplies nuisance free eggs for more than 40 cities nationwide 1500 large supermarkets. But due to the lack of sound mandatory regulations, the director of cooperatives considers to exit "agriculture super-docking” because the supermarkets find all kinds of reasons to charge a fee to cooperatives that make their profit is very low [6].

In order to promote the development of "agriculture super-docking” better, scholars of different fields studied many problems about "agriculture super-docking”. Most of the researches are to discuss the model status and development strategy of "agriculture super docking”, Dinghuan $\mathrm{Hu}$ et al. (2009) made the mode divide into three categories in the early stages of agricultural super-docking development, namely "farmers + farmer professional cooperatives + supermarket”, "farmers + agricultural industrialization leading enterprises + supermarket" and "farmers + planting base + supermarket" [7]; After a lot of scholars to do counter measure research aiming at the problems existing in the implementation of “agricultural super docking”, Yanjie Xu (2012) thought the modernization of cold chain logistics distribution center can effectively resolve the difficulties that “agriculture super docking” work encountered, and shouldn't remove all the intermediate links in production and marketing for the pursuit of "puredocking” [8]. From the perspective of supply chain management process of “agriculture super-docking”, Ruoqi Xie (2015) put forward to construct the fresh food distribution center as the core in supply chain and outsource distribution business, and pointed out it is the most sensible choice based on cost control analysis for the development of "agricultural super-docking” in future to outsource distribution business to the third party logistics enterprises [9]. The Yinan Wang (2014) found the construction of cold chain logistics distribution center in the agriculture super-docking means a large initial investment and high cost during later stage, some third party logistics enterprises who operated independently reduced the proportion of cold chain distribution because of difficulties to profit, it is suggested that the government should create a better condition for the development of cold chain logistics of agricultural products [10]. Wenxiao Gan et al. (2012) explicitly put forward that as the production and sale of agricultural products supervision, government departments should be responsible for the agricultural products quality and safety testing and reporting to establish and improve the responsibility of agricultural products quality and safety standard system when they studied on the agricultural product quality and safety responsibility under the "agriculture super-docking” mode [11]. Gray et al. (2005) believed that product classification and safety standards should be provided as public goods by the government in their study about agricultural product quality classification and food safety assurance [12].

Based on the above research results can be found the primary task to solve development predicament of "agriculture super-docking” in China at present is the construction of large-scale integrated cold chain logistics distribution center. And in the current market environment of "agricultural super-docking” development, the works about quality and safety testing and product grade determination need the "visible hand" of government to adjust, it will be an inevitable choice for many small and medium scale farmer professional cooperatives and supermarkets to rely on a professional third-party cold chain logistics enterprises servicing the docking process of circulation processing and the product transportation as well. Besides, considering the current situation of the development of third-party cold chain logistics enterprise in China, government participation is necessary in capital investment and relationship coordination among multiple subjects. So this paper puts forward the model of "cooperation between the government and the third party logistics enterprises to build distribution center" in agricultural super-docking.

\section{The Model Layout and Expected Impact}

The establishment of "cooperation between the government and the third party logistics enterprises to build dis- 
tribution center" model is based on the "farmers + farmer professional cooperatives + supermarket" docking background, which relates to the subjects are farmer professional cooperatives, government, the third party logistics enterprises, supermarkets and consumers. The overall model layout is shown in Figure 1.

\subsection{Operation Process}

First of all, the product grade requirements, processing standards and transportation requirements of fresh agricultural products should be formulated by supermarkets according to the target customer needs, and provide a detailed description to the cooperation of the farmer professional cooperatives and distribution centers. The government will carry out unified planning for the third party logistics enterprises that participate in this model, they will be classified into different levels as the "service providers" in distribution center according to the present situation of the development of the cold chain facilities level, these service providers will be assigned to provide services for the supermarkets which have the corresponding cold chain requirements. The distribution center will transport agricultural products to the distribution center from professional cooperatives using the cold chain facilities with different conditions. The basic standard of cold chain transportation is formulated and published by the government.

Agricultural products arriving at the distribution center need to carry out a mandatory quality and safety inspection at first, which requires the Ministry of agriculture agricultural product quality and safety center to provide strong support to allow distribution center set up relevant departments and provide the support of professional personnel and equipment. At the same time the real time supervision of government will ensure the validity of the test results, only pass the quality and safety testing, products can enter the circulation processing. In addition, as a value-added service provided by distribution center, product grade certificate service that could be established under the support of Chinese Academy of Agricultural Sciences, China Green Food Association or other related departments can provide paid product level certification to the farmer professional cooperatives, the period of validity of the certificate is determined by the supermarket, the cooperatives need to provide a new proof to the supermarket in the end of the validity of the certificate. The quality and safety testing standards and product grade evaluation system that relate to the above need be formulated and published in detail by the government.



Figure 1. The layout of "cooperation between the government and the third party logistics enterprises to build distribution center" model. 
After the product safety testing, agricultural products will be processed with different standard, from the most simple processing "sorting and packaging combination" to"(pre-packed) clean vegetable” realized directly into the cooking, supermarkets must to pay the appropriate fees corresponding to different levels services.

Finally, agricultural products through the distribution center detection and processing will be transported to the various supermarkets for consumers to buy with the different levels of cold chain transportation.

\subsection{Expected Impact}

The "cooperation between the government and the third party logistics enterprises to build distribution center" model not only inherits the "farmers + farmer professional cooperatives + supermarket" model has fewer intermediate links and lower damage rate, but also can ensure the benefit of the participants.

In this model, farmers can improve their bargaining power in the cooperative negotiation using the authoritative product certification documents provided by the relevant government departments. In addition, the problem that they haven't the specialized logistics and transport facilities and cannot directly with the supermarket standard docking won't make them not dare to involve in agricultural super-docking. Cooperatives only need to concentrate on organizing and training farmers to learn more professional cultivation technology. Improving the level of agricultural products will be expected to get a higher benefit.

Supermarkets not only won't have to pay attention to logistics operations and product standardization, but also can provide customers with more fresh products through the docking with the cooperation in this model. They can grasp the characteristic of instant consumption for fresh products and focus on the supermarket business, so as to compete with peer companies and electricity suppliers better.

The third party logistics enterprises that build the distribution center with the government can use the government's financial support to achieve their own development, on the other hand, the third party logistics enterprises and the government together can get more farmers professional cooperatives and supermarket recognition by providing effective product inspection and standardization of circulation processing services. So as to realize make the large cold chain distribution center as the main body to create the brand attracting more farmers to participate in professional cooperatives and supermarkets, the third party logistics companies will gain the benefits of the brand effect as well.

For the government, the implementation of this model will bring huge social benefits. Distribution center sets up the quality and safety testing process under the government supervision, which will effectively curb the problem that agricultural products of hidden safety risks sold to customers. The government coordinates the third party logistics enterprises and guides them to become bigger and stronger by holding shares, mergers and acquisitions, collaborative alliances, etc. This will effectively integrate social cold chain resources and promote the construction and development of the cold chain logistics infrastructure.

\section{Management Issues in the Model Implementation}

\subsection{The Orientation of the Core Subject in the Agricultural Products Supply Chain}

To make the government and the third party logistics enterprises be able to form a stable supply chain of agricultural products with the farmer professional cooperatives and supermarkets to operate distribution center, there should be a main body as the core. It can organize and coordinates the operation of logistics, information flow and cash flow in the whole supply chain, so as to drive the entire supply chain under the pull of consumer demand. At the beginning of the implementation of "cooperation between the government and the third party logistics enterprises to build distribution center" model, there are restrictions on the other three parties except the government in the capital, scale and other aspects. So in the initial stage the government will be the leading force in the construction of large cold chain distribution center that will coordinate the third party logistics enterprises to form the cold chain transport team and guide the long-term cooperation of farmer professional cooperatives and supermarkets.

But in the long run, the operation of this mode is still to achieve market driven. The third party logistics enterprises will adapt compete in the market in the form of the same industry alliance through the government's long-term support, and will be familiar with the operation characteristics of agricultural products supply chain. So the third party logistics enterprises will assume the functions of the supply chain organizer and manager when the development of the whole third party logistics enterprise organization scale achieves a certain scale. 


\subsection{The Level Division of the Third Party Logistics Enterprises}

When the government is combined with the third party logistics enterprises to build a distribution center in this model, it carries on the division of orders to the supermarket adopting the way of dividing the third party logistics enterprises in order to make full use of the existing cold chain transport resources. This method makes these third party logistics companies bear different the added value of the business, which leads to profit levels are also different. It will inevitably lead to the instability of coordination and cooperation if this situation persists for a long time, so the government needs to assess and adjust the level of these third party logistics enterprises periodically to make those well-developed enterprises can continue to enhance the added value of their own tasks, which plays an effective incentive. Along with the development of these third party logistics enterprises, the distribution center can continue to introduce new third party logistics enterprises to enhance the service quality of distribution center, meanwhile, expand the scale of operation (Figure 2).

\subsection{The Planning Problem of Supermarket's Quantity}

According to the relevant data analysis, an ordinary community supermarket that is the supply of fresh agricultural products won't establish a distribution center until the number of chain stores reaches more than 32 in a city [2]. Because only such a quantity at least can realize the scale effect to offset the logistics and procurement costs, so as not to influence the efficiency and competitiveness of the supermarkets. So in this mode implementation, the problem should be considered. At the early stage of construction, distribution centers need to predict the minimum value that can make the distribution center's operations generate economies of scale based on the cost data of the government and the third party logistics enterprises to build together. If the number of supermarkets does not reach this value in a certain area, the distribution center cannot be established blindly. It should be given priority to continue to improve the degree of participation of supermarkets in this area to increase the degree of customer density. And after the establishment of the distribution center, the continuous expansion of the development scale will make it coming into diseconomies of scale that the long-term average cost starts to increase. The distribution center should stop increase the number of supermarket at this point to avoid profit

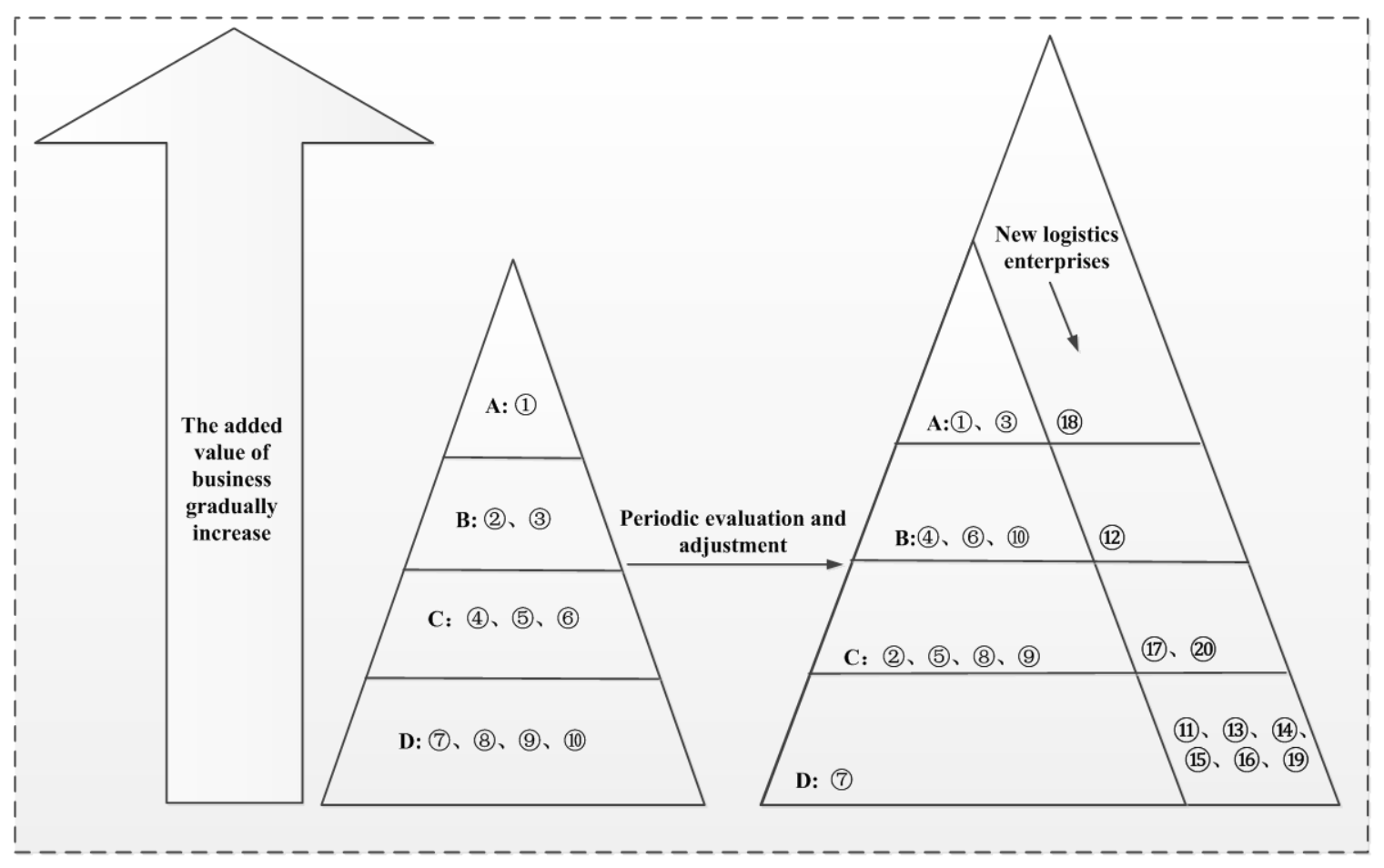

A-D: service level code (1)-(20: The third party logistics enterprise code

Figure 2. Periodic evaluation and adjustment for the third party logistics enterprises. 
decline. The scale effect on long term average cost in the process of development of distribution center is shown in Figure 3.

\subsection{The Problem of Income Distribution among Multiple Subjects}

The operation of the distribution center is the collaboration between the government departments and many third party logistics enterprises in this "cooperation between the government and the third party logistics enterprises to build distribution center" model, so it is needed to develop and implement a reasonable income distribution mechanism to make the operation of the distribution center coordinated and steady. From the overall layout of the model can find that the distribution center can get the benefit of two services, one is to provide product inspection services for farmer professional cooperatives, and the other is logistics services that to provide standardized circulation processing and cold chain transport services for supermarkets and farmers. There into, the incomes of product inspection should be used as the corresponding department personnel salary support, and as a part of the complete integration of the distribution center; the income of circulation processing should be allocated according to the proportion of human and material resources that the third party logistics enterprise investment in during the integrated construction period; the income of cold chain transportation is divided according to the level mentioned above, and the third party logistics company of different level obtains the corresponding level of business profit (Figure 4).

\subsection{The Issue of Supportive Policy Environment}

After implementing the "cooperation between the government and the third party logistics enterprises to build distribution center" model, if there is no good policy environment support, its applicable effect will be weakened greatly. As the most authoritative policy makers in the model proposed in this paper, the government should give the "agriculture super-docking" a good development environment to make it promote further.

First of all, the Ministry of agriculture, Chinese Academy of Agricultural Sciences and other departments should establish the relevant standards for the production and circulation of agricultural products to support farmer professional cooperatives' the implementation of standardized production and carry out grading, packing and labeling of agricultural products. They need strengthen safety record management to achieve the quality of cooperatives products can be traced back [13]. The Ministry of Commerce, Ministry of transport and other departments should also continue to further refine the guidelines about promoting the healthy development of the cold chain transportation and logistics enterprises, so as to better guide cold chain logistics enterprises to improve the efficiency and reduce costs with the integration of resources.

Secondly, farmer professional cooperatives have paid the cost of product safety testing and grading certificate in the model, and agricultural products that passed through the circulation process of large specialized cold chain distribution center which is the combination of the government and the third party logistics can meet the requirements of the standardization of the supermarket. This requires the government needs to truly implement

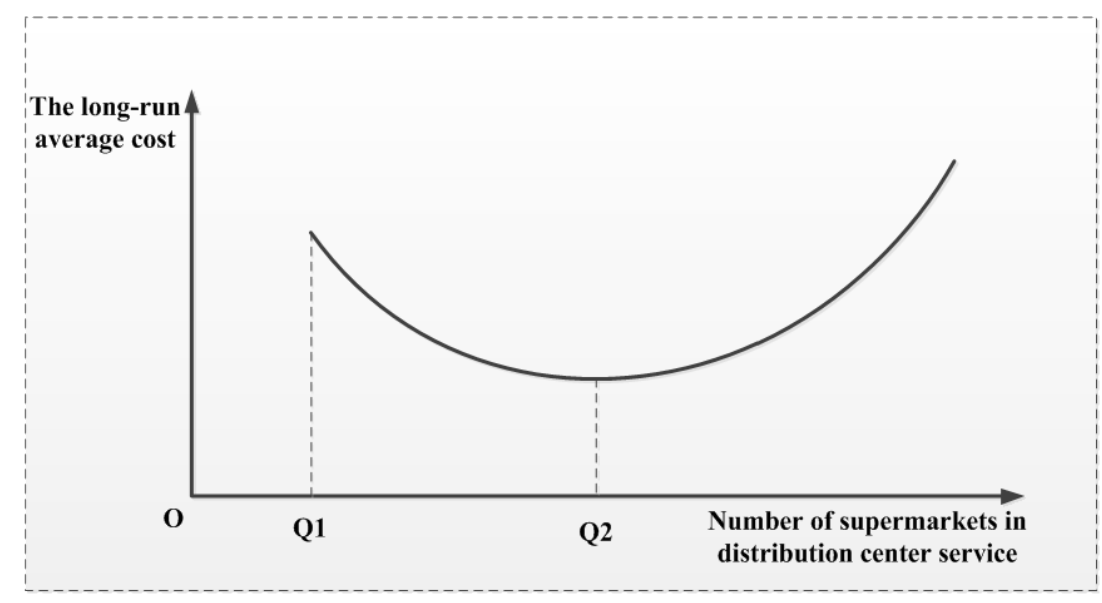

Figure 3. The scale effect in the development of distribution center. 


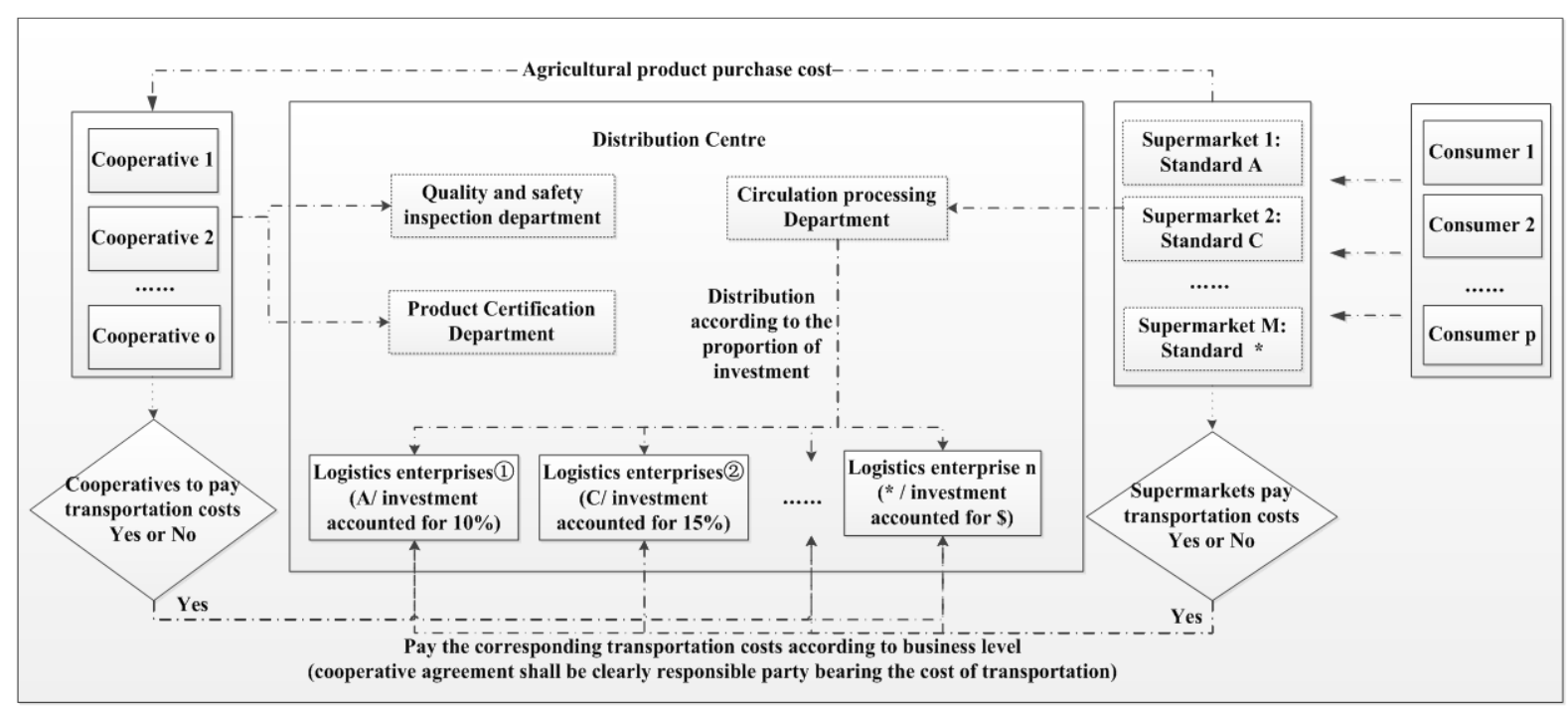

* means (a level between A-D) \$ means a value of the investment share $\quad-\rightarrow$ cash flow

Figure 4. The income distribution among multiple subjects in the distribution center operation.

some of the mandatory provisions after the implementation of the model, for example, it don't allow supermarkets to collect unreasonable charges because of display, bar code, holidays and other reasons and requires the market must give a high degree of acceptance to the relevant certification documents provided by the distribution center, so that farmer cooperatives can get the benefits.

Finally, the government should encourage these entities such as farmer professional cooperatives and supermarket chains to join the "Internet+" tendency in China by providing financial or talent support, which promotes the construction of "agricultural super-docking" management information system that can release of supply and demand information timely, and make "agricultural super-docking" realize online signing and trading gradually.

\section{Conclusion}

There are many circulation models in the implementation of "agriculture super-docking” because the situation of participating subject is different, and all kinds of models have their applicable environment and scope of application. This paper puts forward the "cooperation between the government and the third party logistics enterprises to build distribution center" model based on the "farmers + farmer professional cooperatives + supermarket" docking background in China. This model has two outstanding advantages. One is that the government provides authoritative product testing services as a public goods; the other is that the third party logistics enterprises jointly provide standardized circulation processing services. It is suitable for small and medium sized farmer professional cooperatives, supermarkets and third party logistics that don't have a strong financial strength and a high degree of brand influence to participate in the construction. The model not only inherits that the "farmers + farmer professional cooperatives + supermarket" model has fewer intermediate links and lower damage rate, and protects the interests of the subject of "agriculture super docking", but also solves the problem of quality and safety problems and different standard products in the supply chain, and meanwhile produces considerable social benefits in promoting cold chain infrastructure construction and other aspects. Considering the different development background of "agricultural super-docking" in the process of implementing the model, these aspects about the combination of local natural conditions, economic base and policy environment, etc. should be paid special attention to. To collect detailed data of relevant subjects and deal with management issues about the determination of core subject in supply chain, the method of evaluating the third party logistics enterprises grade, the planning of the number of customers for the distribution center, the mechanism of income distribution among multiple subjects, the implementation of relevant policies after the model into practice and so on ensure full implementing effectiveness of the model. 


\section{References}

[1] Wang, Q.H. (2014) The Misreading and Alternative Models Theory of “Agricultural Super-Docking”. Commercial Times, 6, 16.

[2] Yang, J.Y. and Tang, B.L. (2012) The Present Situation and Problems of Agricultural Super-Docking of Fresh Agricultural Products in China. Jiangsu Agricultural Science, 1, 357.

[3] Wu, W.S. and Chen, J.M. (2013) The Present Situation and Countermeasure of the Development of the Third Party Cold Chain Logistics in China. Logistics Technology, 1, 20.

[4] Wang, X.H. and Zhang, Q.L. (2016) The Inherent Mechanism of E-Commerce Solving Circulation Difficulties of Fresh Agricultural Products-Based on Two Case Comparative Study for the Tmall Fresh and Tuotuo Community. China Soft Science, 2, 50.

[5] Shen, X.J. and Wang, Y. (2013) Analysis of the Operation Mode of Food E-Commerce Platform Based on Quality and Safety. China’s Circulation Economy, 12, 53.

[6] Shuang, K. and Li, J.S. (2015) “Agriculture Super-Docking” Should Dock Really. Chinese Farmer Cooperatives, No. 7104, 22.

[7] Hu, D.H., Yang, W.M. and Zhang, Y. (2009) The Development of “Agricultural Super-Docking” and Farmer Professional Cooperatives. Rural Management, 8, 13.

[8] Xu, Y.J. (2012) Cold Chain Logistics Distribution Center Is an Integral Part of the "Agriculture Super-Docking”. Guangdong Cooperation Economy, 2, 19.

[9] Xie, R.Q. (2015) “Agricultural Super-Docking” Supply Chain Optimization of Fresh Agricultural Products in Shanxi Province. Taiyuan University of Technology.

[10] Wang, Y.N. (2014) Analysis on the Problems and Countermeasures of Cold Chain Logistics Distribution Center under the Background of Agricultural Super-Docking. Technology and Economy, 1, 47.

[11] Gan, W.X. and Liu, X.B. (2012) Research on Agricultural Product Quality and Safety Responsibility System under the Mode of “Agricultural Super-Docking”. Agricultural Economy, No. 30106, 124.

[12] Gray, R.S., Richard, S., Sumne, D.A., Alston, J.M., Brunke, H. and Acquaye, A.K.A. (2005) Economic Consequences of Mandated Grading and Food Safety Assurance: Ex Ante Analysis of the Federal Marketing Order for California Pistachios. CA: Giannini Foundation Monograph 46, March.

[13] Liu, J.S. (2011) What Are Policies to Support “Agricultural Super-Docking”. Guide of Sci-Tech Magazine, 28, 16.

\section{Submit or recommend next manuscript to SCIRP and we will provide best service for you:}

Accepting pre-submission inquiries through Email, Facebook, LinkedIn, Twitter, etc.

A wide selection of journals (inclusive of 9 subjects, more than 200 journals)

Providing 24-hour high-quality service

User-friendly online submission system

Fair and swift peer-review system

Efficient typesetting and proofreading procedure

Display of the result of downloads and visits, as well as the number of cited articles

Maximum dissemination of your research work

Submit your manuscript at: http://papersubmission.scirp.org/ 